\title{
Evaluación de corredores biológicos en Costa Rica: estructura de paisaje y procesos de conectividad- fragmentación
}

\section{Assessment of biological corridors in Costa Rica: landscape structure and connectivity-fragmentation processes}

\author{
Carlos Morera-Beita ${ }^{l}$ \\ Universidad Nacional, Costa Rica \\ Luis Fernando Sandoval-Murillo ${ }^{2}$ \\ Universidad Nacional, Costa Rica \\ Luis Diego Alfaro-Alvarado \\ Universidad Nacional, Costa Rica
}

\begin{abstract}
Resumen
En el planeta, durante los últimos años, los corredores biológicos han proliferado como respuesta al proceso acelerado de fragmentación de los ecosistemas naturales. Basada en esta estrategia, Costa Rica desde la década de 1990, ha implementado corredores biológicos como espacio de conectividad de las áreas protegidas que funcionan como paso entre áreas núcleo. Durante los últimos años, se han establecido 40 corredores biológicos que cubren el 38\% de la superficie de Costa Rica. A pesar de su popularidad, no existen investigaciones que evalúen su funcionalidad como espacios de conectividad. Basada en esta necesidad, este artículo analiza los corredores biológicos de Costa Rica desde la perspectiva de: área, estructura del paisaje, funcionalidad ecológica y niveles de conectividad-fragmentación. Se utiliza la base de datos del Programa Nacional de Corredores y se

1 Escuela de Ciencias Geográficas, Universidad Nacional (UNA), Costa Rica.

Correo electrónico: cmorera@una.cr. (D) https://orcid.org/0000-0002-4014-6122

2 Escuela de Ciencias Geográficas, Universidad Nacional (UNA), Costa Rica.

Correo electrónico:

luis.sandoval.murillo@una.cr . (D) https://orcid.org/0000-0002-8325-1117

3 Instituto Internacional en Conservación y Manejo de Vida Silvestre (ICOMVIS), Universidad Nacional (UNA), Costa Rica. Correo electrónico: luis.alfaro.alvarado@una.cr.

(iD https://orcid.org/0000-0001-9534-1948
\end{abstract}


complementa con análisis de coberturas usando imágenes Landsat del 2010 y 2015. Se concluye que los corredores biológicos, en Costa Rica son diversos en áreas, formas, como funcionalidad ecológica y niveles de conectividad-fragmentación. Posterior a su establecimiento la mayoría de los corredores biológicos han recuperado parte importante de la cobertura natural, sin embargo la fragmentación se ha incrementado. La abundancia de corredores biológicos evidencia una falta de política de priorización, que es fundamental para avanzar en la consolidación de estas estrategias de conectividad en el país.

Palabras clave: Corredores Biológicos, Áreas Protegidas, conectividad, Costa Rica.

\begin{abstract}
In recent years, biological corridors have proliferated worldwide in response to the accelerated process of fragmentation of natural ecosystems. Since the early 1990s, Costa Rica implemented the establishment of biological corridors as a strategy for seeking connectivity among protected areas. During the past years, forty new biological corridors have been established, equivalent to $38 \%$ of the total surface area of Costa Rica's mainland territory. Despite their popularity, there is no research evaluating their functionality as connectivity spaces. In view of this shortcoming, the present article analyzes Costa Rica's biological corridors from the perspectives of area, landscape structure, ecological functionality, and levels of connectivity-fragmentation. The database of the Programa Nacional de Corredores (translated as National Program of Corridors) is used and complemented by coverage analysis using Landsat images from 2010 and 2015. It is concluded that biological corridors in Costa Rica are diverse in regards to area coverage, landscape structure, ecological functionality and degree of connectivity-fragmentation. After their establishment, most biological corridors have recovered an important part of their natural coverage, nonetheless, fragmentation has increased. The abundant existence of biological corridors evidences the lack of a prioritization policy essential to advance the consolidation of these connectivity strategies throughout the country.
\end{abstract}

Keywords: Biological corridors, Protected areas, Connectivity, Costa Rica.

\title{
Introducción
}

El antropoceno como época geológica, está determinado por la influencia de las actividades humanas en los ciclos biogeoquímicos del planeta (Lewis y Maslin, 2015), siendo el cambio climático uno de los factores que contribuye a la fragmentación de los ecosistemas naturales (Fung, Imbach, Corrales, Vílchez, Zamora, Argotty \& Ramos, 2017).

En la actualidad, la preservación y la restauración de la conectividad en los ecosistemas naturales es uno de los principales desafíos para la conservación de la biodiversidad y la planificación del paisaje (Ersoy, Jorgensen \& Warren, 2018), especialmente en los escenarios tropicales como Costa Rica (Taubert, Fischer, Groeneveld, Lehmann, Müller, Rödig \& Huth, 2018; Morera y Sandoval, 2016). 
Durante el siglo pasado, las investigaciones sobre el cambio climático y las condiciones de biodiversidad identificaron que las áreas protegidas son islas aisladas de áreas silvestres que necesitan ser conectadas (Worboys, Francis \& Lockwood, 2010). Así emergen los corredores biológicos (CB), como espacios que facilitan el movimiento de especies (flora y fauna) y se fundamentan en observaciones teóricas y empíricas sobre cómo el intercambio de individuos entre las poblaciones puede posibilitar la persistencia particularmente para tamaños de poblaciones menores al mínimo viable según el grupo taxonómico, el cual puede inclusive requerir varios miles de individuos (Lochran, Traill, Corey, Bradshaw \& Brook; 2007) y aisladas (Rosenberg, Noon \& Meslow; 1997). Por otro lado, algunos autores plantean los efectos adversos de los CB como facilitadores de dispersión de plagas y enfermedades (Fletcher, Didham, Banks-Leite, Barlow, Rosindell y Melo, 2018).

Durante las últimas décadas, se establecieron los CB con diversos tamaños y estructuras de paisajes, condiciones socioeconómicas así como características de la cobertura natural, transformándose en una de las estrategia de mayor relevancia a nivel del paisaje para la conservación de la biodiversidad (Hilty, Arnfalk, Erdmann, Goodman, Lehmann y Wäger, 2006).

En 1994, el estado costarricense capitalizando la experiencia del Servicio Nacional de Parques fundando durante la década de los 70's y con el objetivo de preservar la biodiversidad, se estableció el Sistema de Parques Nacionales, el cual en los 90's se convierte en el Sistema Nacional de Áreas de Conservación (SINAC), oficializando las siguientes categorías: reservas biológicas, parques nacionales, refugios de vida silvestre, zonas de protección, reservas forestales y humedales (Morera y Nel-lo, 2017).

No obstante, los CB's no se consideran una categoría oficial de conservación, debido a que son espacios que incluyen propiedad privada y pública, donde se procura la conectividad ecológica aunado a esfuerzos de gestión productiva y de conservación. Con el objetivo de gestionar los CB's se estableció el Programa Nacional de Corredores Biológicos (PNCB) en 2006 (Villate, Canett, Chassot y Monge, 2009), que determinó estas áreas como prioritarias para la asignación de Pago por Servicios Ambientales. De acuerdo con la reglamentación nacional para aprobarse un $\mathrm{CB}$ deben registrarse un consejo local, así como presentar un perfil 
técnico y proponer un plan estratégico, avalado por el CORAC (Consejo Regional de Área de Conservación) y aprobado respectivamente por el CONAC (Consejo Nacional de Áreas de Conservación).

Los antecedentes de los CB's son anteriores al establecimiento del PNCB. En Costa Rica, a fines de la década de 1990 por iniciativa de una organización no gubernamental se establece el Corredor Biológico Talamanca-Caribe, siendo el primero en el país y que fue un antecedente para la posterior definición del Corredor Mesoamericano en la costa caribeña de América Central (Solís, Madrigal, Cruz y Fonseca; 2002).

En 2018, Costa Rica cuenta con 44 corredores que cubren el 38\% de la superficie del país y han sido razón de diversas investigaciones como: Acuña, Molina y Rodríguez (2017); Chinchilla, (2015); Bosselmann, (2012); Villate et al., (2009); Alarcón, Anzueto, Galindo, García, Gómez, Porras y Sáenz, (2003), entre otros.

La mayoría de estos estudios se centran en evaluar un $\mathrm{CB}$, mientras que solo existe un estudio realizado por Calvo-Obando, (2009) que analiza las condiciones de conectividad de los CB's en el país basado en algunos índices de paisaje.

Fundamentado en la necesidad de valorar los CB's del país, este artículo plantea como objetivo evaluar los CB's considerando la superficie, algunos índices relacionados con la estructura del paisaje (Número, tamaño medio y desviación estándar de los fragmentos, la función ecológica, los procesos de fragmentación-conectividad) y su localización de acuerdo con los pisos altitudinales en Costa Rica para determinar su funcionalidad como espacios de conectividad. Para lo anterior se aplica el enfoque de la Ecología del Paisaje (Moss, 2001); específicamente la escuela norteamericana fundamentada en el modelo Matriz-Parche-Corredor (Forman, 2014).

\section{Área de estudio}

Costa Rica se localiza en el istmo centroamericano, que enlaza la región neotropical (Sudamérica) con la neártica (Norteamérica), permitiendo históricamente migraciones de flora y fauna en ambas direcciones (Kappelle, 2016). Esta conectividad, agregado a un amplio rango de elevaciones así como la diversidad climática, geomorfológica y edafológica, ha generado una región con alta variación ecológica a pesar de su limitada área $\left(51,100 \mathrm{~km}^{2}\right)$. 
Actualmente el país alberga alrededor del $5 \%$ de la biodiversidad del mundo según Criado-Hernández y Marín-Cabrera (2008) y acoge diferentes ecosistemas como: páramo, bosque tropical lluvioso, bosque seco tropical, manglares, sabana, bosque inundado, humedal, entre otros.

La biodiversidad terrestre está resguardada por medio de 148 espacios protegidos ( $25 \%$ del territorio continental del país), de los cuales los parques nacionales $(\mathrm{n}=33)$ cubren el $12 \%$, y las reservas biológicas $(\mathrm{n}=$ 8 ) el $0,4 \%$ cuyo uso es exclusivamente para la conservación y protección del ecosistema (Cuadro 1).

Existen otras categorías que incluyen territorios privados, públicos o mixtos tales como; refugio de vida silvestre, reserva forestal, zona protectora y humedal nacional, conjuntando un total de 58 espacios protegidos (8.9\% del espacio terrestre país).

En el establecimiento y la gestión de áreas protegidas como estrategia para la conservación de la biodiversidad terrestre, Costa Rica desarrolló un sistema de corredores biológicos y ha declarado por decreto 44 CB's que cubren $38 \%$ de la superficie del país (Figura 1).

\section{Cuadro 1. Categorías de las Áreas Silvestres Protegidas y Corredores} Biológicos en Costa Rica, 2018

\begin{tabular}{|c|c|c|c|c|}
\hline $\begin{array}{c}\text { Condición de la } \\
\text { propiedad }\end{array}$ & Categoría & Número & Área (ha.) & $\begin{array}{c}\text { \% Área de } \\
\text { país }\end{array}$ \\
\hline \multirow{4}{*}{ Estatales } & Reserva Biológica & 8 & 21432 & 0.4 \\
\cline { 2 - 5 } & Parques Nacionales & 28 & 587800 & 11.4 \\
\cline { 2 - 5 } & Sub-Total & 33 & 589232 & 11.8 \\
\hline \multirow{4}{*}{ Estatales y privadas } & $\begin{array}{c}\text { Refugio de Vida } \\
\text { Silvestre }\end{array}$ & 47 & 175524 & 3.4 \\
\cline { 2 - 5 } & Reserva Forestal & 11 & 284133 & 5.5 \\
\cline { 2 - 5 } & Sub-total. & 58 & 459657 & 8.9 \\
\hline \multirow{4}{*}{ Privadas } & Zona Protectora & 31 & 157213 & 3.0 \\
\cline { 2 - 5 } & Humedal Nacional & 14 & 69251 & 1.3 \\
\cline { 2 - 5 } & Otras & 13 & 21811 & 0.4 \\
\cline { 2 - 5 } & Sub-total & 58 & 273877 & 4.7 \\
\hline TOTAL & & 148 & 1322818 & 25.4 \\
\hline Estatales y privadas & Corredores Biológicos & 44 & 1902674 & 38 \\
\hline
\end{tabular}

Fuente: Pfaff, A. S., \& Sánchez-Azofeifa; G. A. (2004); Morera C y Nel-lo M. 2017. http://www.sinac.go.cr/ES/asp/Paginas/default.aspx 
Figura 1. Mapa de los Corredores Biológicos, Costa Rica, 2018

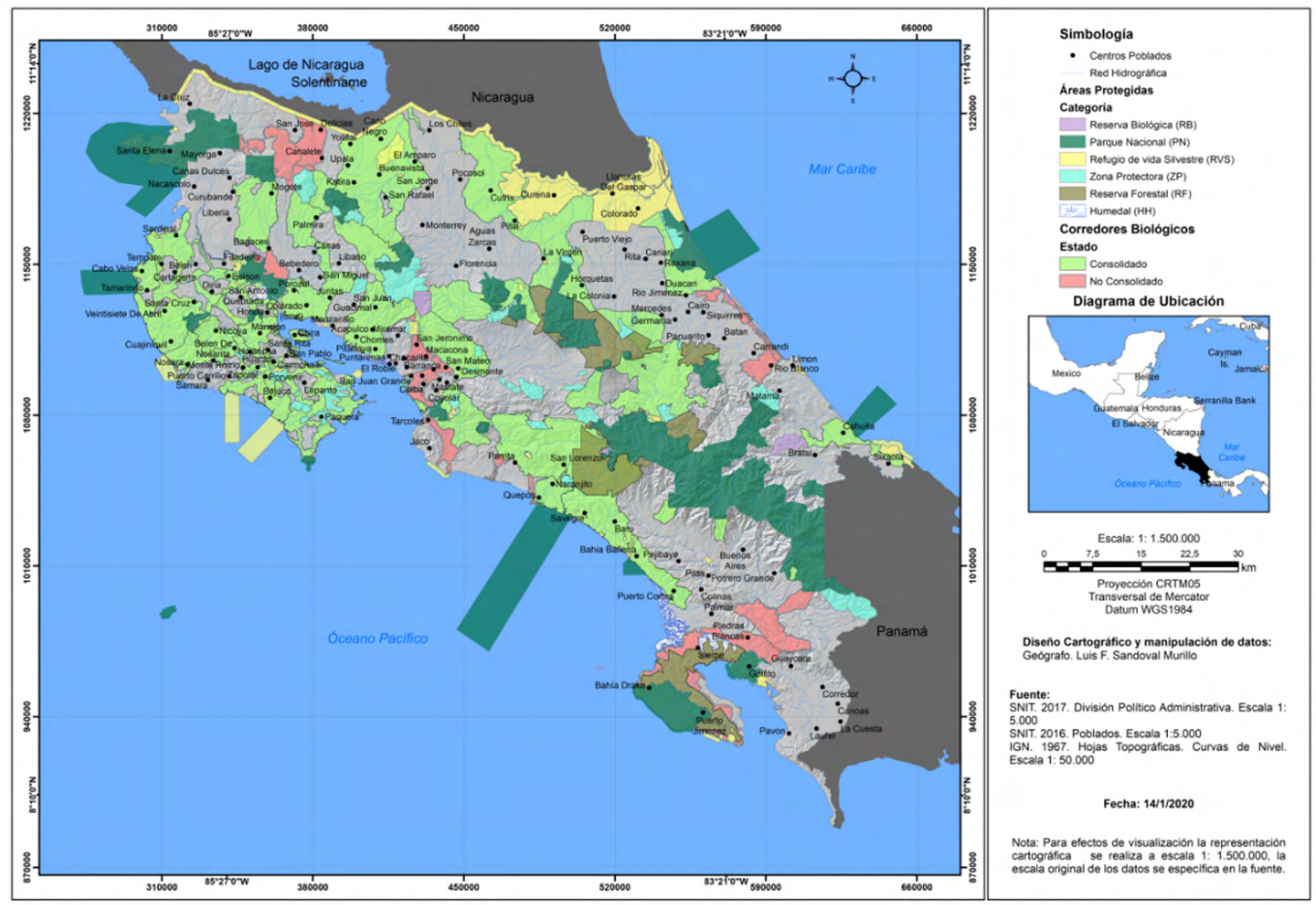

Fuente: Elaboración propia a partir de datos de Programa Nacional de Corredores Biológicos.

\section{Metodología}

Se utilizó una base de datos vectorial administrada por el PNCB para definir los límites de cada CB y se corrigieron los límites de los polígonos a partir de procesos de edición utilizando el programa Arc-Map 10.5. Además, se elaboraron capas de uso de la tierra para los años 2000 y 2015 utilizando imágenes de satélite LandSat7 y LandSat8 con una resolución espacial de $30 \mathrm{~m}$. Asimismo, el programa SIG (Arc-Map 10.5) se utilizó para analizar los CB's y su distribución en los diferentes pisos altitudinales. Los índices de paisaje (número, media y desviación estándar de los fragmentos y vecino próximo) de la cobertura natural para cada $\mathrm{CB}$ fueron calculados usando la extensión Patch Analyst para ArcGIS (Rempel, Carr \& Elkie. 2008). 
Para evaluar las superficie de los CB's se agruparon en tres categorías: micro-local ( 0 - 25,000 ha), local (de 25,001 a 50,000 ha) y regional (más de 50,001 ha). La estructura del paisaje se analizó basándose en tres abordajes: el primero consideraba un $\mathrm{CB}$ como una franja o banda donde las especies se mueven sin direcciones específicas (Hilty et al, 2019), el segundo lo concibe como una red donde las especies pueden moverse en direcciones específicas (IBID) y el último como piedra-escalón (steppingstone) donde las especies se trasladan de un fragmento a otro (DuforetFrebourg, \& Slatkin, 2016), (Figura 2). Desde este enfoque las estructuras paisajísticas pueden ser fundamentalmente de dos tipos: redes y franjas, los cuales se relacionan con los conceptos de piedra-escalón o sea espacios discontinuos o continuos.

Figura 2. Enfoque de estructura de paisaje dominante para el diseño de corredores biológicos.

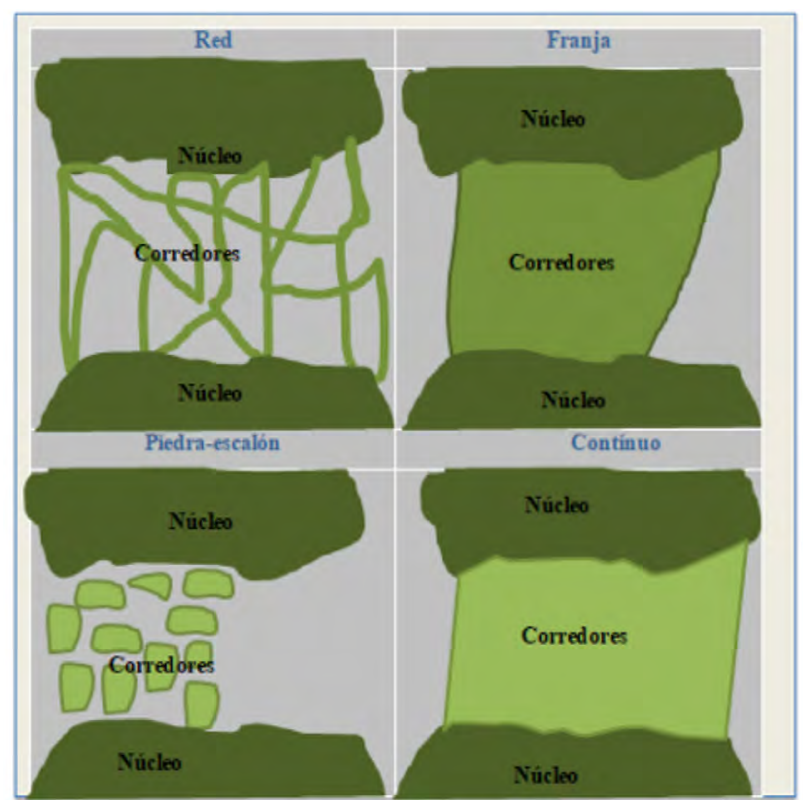

Fuente: adaptado de Forman (2014) y Hilty et al, (2019).

La función ecológica del CB se clasificó en cuatro tipos: corredores como zona de amortiguamiento (de Lima, Moura, da Costa, de Lima, de Melo Lucena, Crispim, y de Souza, 2019), (extensión de un fragmento 
natural que se preserva como extensión de un área núcleo pero sin conexión con otro), corredor conectado (mantiene el $60 \%$ de la cubierta natural del corredor), corredor fragmentado (vegetación natural que representa menos del $60 \%$ de la conexión paisaje) y el corredor ribereño y costero (se utilizan ecosistemas ribereños y costeros como conectores) (Hilty et al, 2019).

Para evaluar los cambios en la cobertura natural de los diferentes períodos de estudio, se calculó la tasa de cambio de la vegetación natural de acuerdo a la fórmula propuesta por la FAO (1996):

$$
\mathrm{C}=\left((\mathrm{T} 2 / \mathrm{T} 1)^{(1 / \mathrm{n}-1) *} 100\right.
$$

Dónde:

$$
\begin{aligned}
& \mathrm{C}=\text { Tasa de cambio de la cobertura natural } \\
& \mathrm{T} 1=\text { Año de inicio (con el que se quiere comparar) } \\
& \mathrm{T} 2=\text { Año actual o más reciente } \\
& \mathrm{n}=\text { Número de años entre T1 y T2 }
\end{aligned}
$$

La dinámica de la cobertura natural se analizó mediante el índice de fragmentación-conectividad (IFC), utilizando la ecuación adaptada por Morera y Sandoval (2018) para la cobertura natural de cada CB en el período de estudio.

IFC:

$$
\frac{\text { SPTA x } 100}{\operatorname{Sm} \times\left(\sum \mathrm{Nm} / \mathrm{Dm}\right)}
$$

Donde:

IFC: índice de fragmentación - conectividad

SPTA: superficie (ha) del corredor biológico.

Nm: Número de fragmentos de cobertura natural.

Sm: Superficie de fragmentos de cobertura natural.

Dm: Distancia promedio de la cobertura natural calculada desde el centro de cada una.

\section{Resultados y discusión \\ Tamaño de los CB's}

Los CB's presentan una amplia diversidad de tamaños, formas y funcionalidades en Costa Rica. Existe una heterogeneidad en a cuanto superficie, los corredores micro-locales cubren $9.6 \%$ del área total mientras que los regionales representan $71.5 \%$ (Figura 3). 
Dentro de los requerimientos oficiales para establecimiento de un $\mathrm{CB}$, el tamaño del área no es un elemento relevante y los límites se modifican cuando el comité local lo apruebe y lo proponga al CORAC para posteriormente ser refrendado por el CONAC, sin requerir un estudio técnico pertinente que los justifique.

Figura 3. Corredores Biológicos de Costa Rica: categoría de superficies, 2018

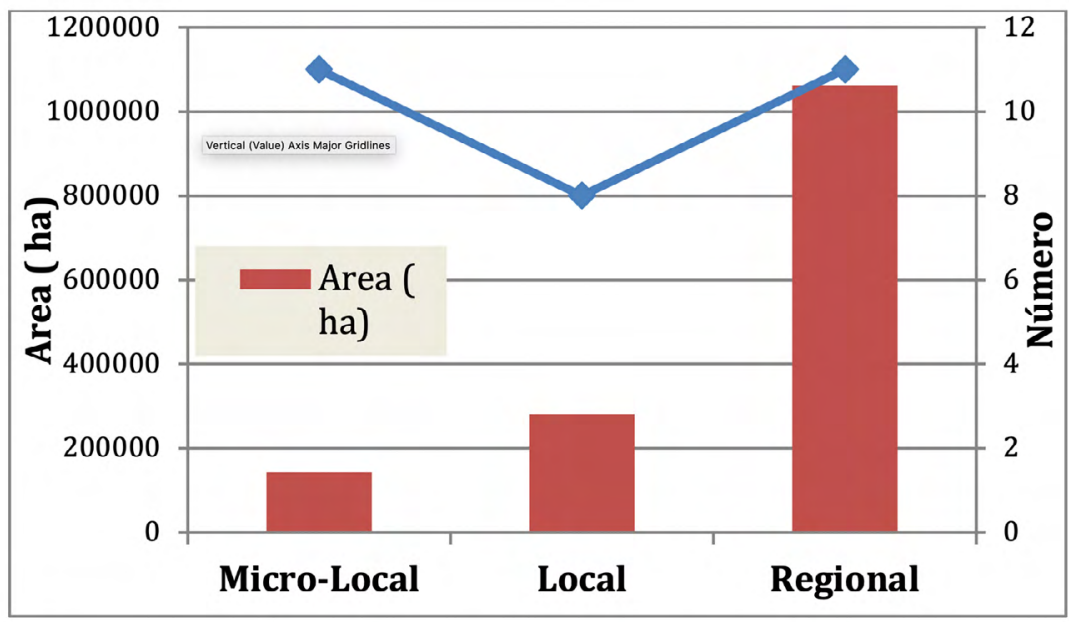

Fuente: elaboración propia

La superficie de un corredor biológico es determinante, debido a que es un espacio de tránsito de especies, el cual es seleccionado por animales y plantas en función de su forma, longitud, ancho, tipo de hábitats, entre otros (McClure, Hansen \& Inman; 2016), sin embargo, estas valoraciones no se analizan dentro del perfil técnico requerido para la aprobación de un CB.

Por lo tanto, existen algunos $\mathrm{CB}$ con importantes diferencias de superficie como el San Juan-La Selva (1748002 ha), que se extiende desde la Cordillera Volcánica Central hasta la ribera del río San Juan en comparación con el Corredor Los Quetzales-Tres Colinas que cubre únicamente 19370 ha (Figura 4), presentando una diferencia en superficie de $90 \%$.

La heterogeneidad de la superficie de los CB se debe a que para la definición de sus límites no se requiere expropiar, pues su estrategia se 
centra en acciones con la sociedad civil centrados en la gestión del territorio. Igualmente, algunos $\mathrm{CB}$ que se localizan en espacios transfronterizos que obligan a coordinar las acciones con otros países, como el caso del San Juan-La Selva, que conecta con Nicaragua.

Figura 4. Corredores Biológicos en Costa Rica: Categoría de las áreas para 2018

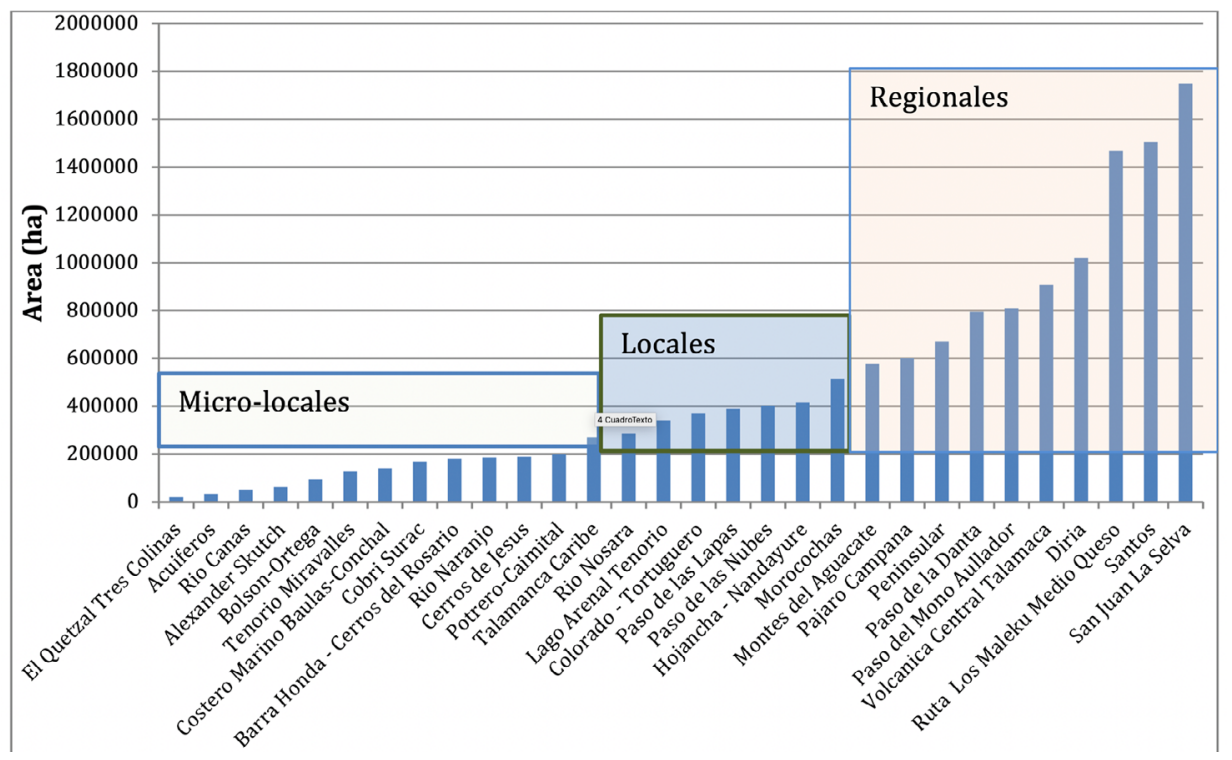

Fuente: elaboración propia

El tamaño apropiado de un $\mathrm{CB}$, y su definición está determinado por las características ecológicas de las especies clave que se conservan o se relacionan dentro de este espacio, sin embargo la definición de hábitat óptimo en cuanto conectividad (Erns 2014, Keeley, Beier \& Gagnon; 2016) y tamaño (Horskins, Mather, \& Wilson, 2006; Vergara 2011) aún es debatida por la ciencia.

El movimiento de los animales es necesario para predecir las tendencias en la distribución de especies y su ensamblaje (Khazan, 2014), así como en la planificación de la conservación, factores que no se consideran de forma precisa dentro del establecimiento de CB en Costa Rica. 
Carlos Morera-Beita Luis Fernando Sandoval-Murillo Luis Diego Alfaro-Alvarado

Evaluación de corredores biológicos en Costa Rica: estructura de paisaje y

procesos de conectividad-fragmentación.

\section{Estructura de los corredores biológicos}

La estructura del paisaje para la conservación ecológica es un mosaico de hábitats con diversidad de escalas y granos (Buzai, Humacata, Lanzelotti, Montes y Principi, 2019) que potencian o limitan los ciclos bio-geoquímicos y con ello preservar la biodiversidad (Morera y Sandoval, 2015).

Por lo cual, las condiciones del hábitat de cada espacio están relacionadas con la estructura paisajística que afecta al movimiento y composición de las especies (Yang, Liu, Zhang, Zeng, Pan, Li \& Lu, 2019).

Considerando la estructura del paisaje, la mitad de los CB's integran una red con otras áreas protegidas, especialmente parques nacionales y reservas biológicas. Existen algunos casos donde los CB son articulaciones con otros espacios de conectividad como son Acuíferos y Montes del Aguacate.

Lo anterior, se respalda en el abordaje de piedra-escalón, donde la vegetación natural no es una ruta continua y las especies de fauna pueden moverse por diversas rutas (Duforet-Frebourg y Slatkin, 2016), por lo cual no se identifican rutas precisas para las especies que habitan o se trasladan en el CB.

Se encontró que 10 de los CB's tienen forma de banda o franja y tres presentan parcialmente esta forma. Ninguno de los CB's integra una red completa y el $57 \%$ articulan con otras categorías de conservación formando una red (Cuadro 2).

La dominancia de un enfoque, en que los CB se conforman articulando con otras categorías de conservación es un indicador de que estos son espacios más regionales y de mayor escala espacial, que obliga a coordinar acciones con otros entes u organizaciones para su debida consolidación y gestión. 
Cuadro 2. Corredores biológicos en Costa Rica de acuerdo a enfoque paisajístico, 2018

\begin{tabular}{|c|c|c|c|}
\hline $\begin{array}{l}\text { Enfoque } \\
\text { paisajístico }\end{array}$ & Número & Área (ha) & Corredores biológicos \\
\hline Banda & 10 & 283210 & $\begin{array}{c}\text { Cobri Surac, Río Cañas, El Quetzal, Lago } \\
\text { Arenal-Tenorio Tres Colinas, Alexander Skutch, } \\
\text { Colorado, Morocochas, Tortuguero, Volcánica } \\
\text { Central_Talamaca, Tenorio_Miravalles, } \\
\text { Talamanca Caribe. }\end{array}$ \\
\hline $\begin{array}{l}\text { Parte de una } \\
\text { banda }\end{array}$ & 3 & 361585 & $\begin{array}{c}\text { San Juan La Selva, Ruta Los Malecu-Medio } \\
\text { Queso, Paso de Las Nubes. }\end{array}$ \\
\hline Red & 0 & 0 & \\
\hline Parte de una red & 17 & 840822 & $\begin{array}{c}\text { Los Santos, Diriá, Paso del Mono Aullador, } \\
\text { Paso de la Danta, Acuíferos, Peninsular, Pájaro } \\
\text { Campana, Montes del Aguacate, Hojancha } \\
\text { - Nandayure, Paso de las Lapas, Río Nosara, } \\
\text { Potrero-Caimital, Cerros de Jesús, Río Naranjo, } \\
\text { Barra Honda - Cerros del Rosario. }\end{array}$ \\
\hline
\end{tabular}

Fuente: elaboración propia.

\section{Función ecológica de los Corredores Biológicos}

Los CB son espacios de conectividad que pueden ser espacios continuos o discontinuos, entre fragmentos y áreas núcleos (Horskins et al., 2006), los cuales serían los parques nacionales o las reservas biológicas, debido a su relevancia como reductos de biodiversidad en el caso de Costa Rica.

Esta investigación identificó que 53\%, son iniciativas en espacios fragmentados, fundamentado en el concepto de piedra-escalón, mientras $17 \%$ se localizan sobre vegetación natural continua.

Siguiendo el enfoque de corredor ripario (Stewart, Wang, Lyons, Horwatich y Bannerman, 2001) o costero se categorizaron únicamente 3 $(10 \%)$. Por otro lado, se destaca que con el objetivo de incrementar las acciones de conservación sobre las áreas de influencia de las áreas núcleos o por confusión teórica, seis CB (20\%) son exclusivamente zonas de amortiguamiento (Cuadro 3). 
Carlos Morera-Beita Luis Fernando Sandoval-Murillo Luis Diego Alfaro-Alvarado

Evaluación de corredores biológicos en Costa Rica: estructura de paisaje y

procesos de conectividad-fragmentación.

Cuadro 3. Corredores biológicos en Costa Rica de acuerdo a su función ecológica, 2018

\begin{tabular}{|c|c|c|c|}
\hline $\begin{array}{c}\text { Función ecológica } \\
\text { del CB }\end{array}$ & Número & $\begin{array}{l}\text { Área } \\
\text { (ha) }\end{array}$ & Corredor Biológico \\
\hline $\begin{array}{l}\text { Corredor de } \\
\text { conectividad }\end{array}$ & 5 & 216700 & $\begin{array}{l}\text { Paso de las Lapas, Paso de Las Nubes, } \\
\text { Lago Arenal-Tenorio, Volcánica Central_ } \\
\text { Talamaca and Tenorio_Miravalles. }\end{array}$ \\
\hline $\begin{array}{l}\text { Corredor so- } \\
\text { bre espacio } \\
\text { fragmentado }\end{array}$ & 16 & 992174 & $\begin{array}{l}\text { Barra Honda - Cerros del Rosario, Diriá, } \\
\text { Paso del Mono Aullador, Río Naranjo, } \\
\text { Potrero-Caimital (Xinia Campos), Río } \\
\text { Nosara, Hojancha - Nandayure, Montes } \\
\text { del Aguacate, Morocochas, Pájaro Cam- } \\
\text { pana, Peninsular, Paso de la Danta, Ruta } \\
\text { Los Malecu-Medio Queso, San Juan - La } \\
\text { Selva, Cobri Surac, Talamanca Caribe. }\end{array}$ \\
\hline $\begin{array}{c}\text { Corredor ripario y } \\
\text { costero }\end{array}$ & 3 & 43017 & $\begin{array}{c}\text { Cerros de Jesús, Costero Marino Baulas - } \\
\text { Conchal and Bolsón-Ortega. }\end{array}$ \\
\hline $\begin{array}{l}\text { Zona de } \\
\text { Amortiguamiento }\end{array}$ & 6 & 233728 & $\begin{array}{l}\text { Acuíferos, Los Santos, El Quetzal Tres } \\
\text { Colinas, Río Cañas, Alexander Skutch } \\
\text { and Colorado Tortuguero. }\end{array}$ \\
\hline
\end{tabular}

Fuente: elaboración propia

\section{Corredores biológicos y pisos altitudinales}

Dentro de la definición de $\mathrm{CB}$, está implícito que este espacio funciona como vía de tránsito para la fauna (Hilty et al., 2019) y que son propuesots para reducir los efectos adversos del cambio climático en la biodiversidad (Heller \& Zavaleta, 2009), lo cual se relaciona con el énfasis en la conectividad de las estrategias de conservación a medida que aumentan las temperaturas globales o regionales (Krosby, Tewksbury, Haddad \& Hoekstra, 2010). Por lo anterior, el establecimiento de corredores biológicos debe incorporar la mayor cantidad de pisos altitudinales, los cual es indispensable en un país que fundamenta parte de su alta biodiversidad en el gradiente ecológico que domina en el territorio. De tal forma, que ese espacio delimitado como CB, permitirá que las especies se muevan facilitando la migración requerida como parte de la adaptación al cambio climático. 
De los cinco tipos de pisos altitudinales (Basal, Pre-montano, montano bajo, montano y subalpina) existente en Costa Rica (García y Gálvez, 2003), solo dos contienen un gradiente ecológico que incluye cuatro pisos altitudinales siendo estos: Volcánica Central-Talamanca y Santos, 8 comprenden 3 pisos altitudinales, 12 se distribuyen en 2 pisos altitudinales y 8 en apenas uno (Cuadro 2; Figuras: 4 y 5).

Cuadro 2. Localización de los Corredores Biológicos de Costa Rica según pisos altitudinales

\begin{tabular}{|c|c|c|}
\hline $\begin{array}{l}\text { Localización en Pisos } \\
\text { Altitudinales }\end{array}$ & Cantidad & Corredor Biológico \\
\hline Basal & $7(23 \%)$ & $\begin{array}{c}\text { Barra Honda- Cerros del } \\
\text { Rosario,Bolsón-Ortega } \\
\text { Cerros de Jesús, Colorado - Tortuguero } \\
\text { Costero Marino Baulas-Conchal, Río Naranjo } \\
\text { Talamanca - Caribe. }\end{array}$ \\
\hline Basal - Premontano & $\begin{array}{c}10(30 \\
\%)\end{array}$ & $\begin{array}{c}\text { Diria, Hojancha - Nandayure, Morocochas, } \\
\text { Pajaro Campana, Paso del Mono Aullador, } \\
\text { Peninsular } \\
\text { Potrero-Caimital, Rio Nosara, Ruta Los Male- } \\
\text { ku Medio Queso, Tenorio Miravalles }\end{array}$ \\
\hline $\begin{array}{c}\text { Basal - Premontano - } \\
\text { Montano Bajo }\end{array}$ & $8(27 \%)$ & $\begin{array}{c}\text { Acuíferos, Alexander Skutch, Lago Arenal } \\
\text { Tenorio } \\
\text { Montes del Aguacate, Paso de la Danta,Paso } \\
\text { de las Lapas, Paso de las Nubes, San Juan - La } \\
\text { Selva. }\end{array}$ \\
\hline $\begin{array}{c}\text { Premontano - Montano } \\
\text { Bajo }\end{array}$ & $\begin{array}{l}1(0.3 \\
\%)\end{array}$ & El Quetzal Tres Colinas \\
\hline $\begin{array}{c}\text { Premontano - Montano } \\
\text { Bajo - Montano }\end{array}$ & $2(0.6 \%)$ & Cobri Surac, Río Cañas \\
\hline $\begin{array}{l}\text { Basal - Premonta- } \\
\text { no - Montano Bajo } \\
\text { - Montano }\end{array}$ & $2(0.6 \%)$ & Santos, Volcánica Central Talamanca \\
\hline
\end{tabular}

Fuente: elaboración propia 
Carlos Morera-Beita Luis Fernando Sandoval-Murillo Luis Diego Alfaro-Alvarado

Evaluación de corredores biológicos en Costa Rica: estructura de paisaje y

procesos de conectividad-fragmentación.

Figura 5. Mapa de los Corredores Biológicos de Costa Rica:

Localización de acuerdo a pisos altitudinales

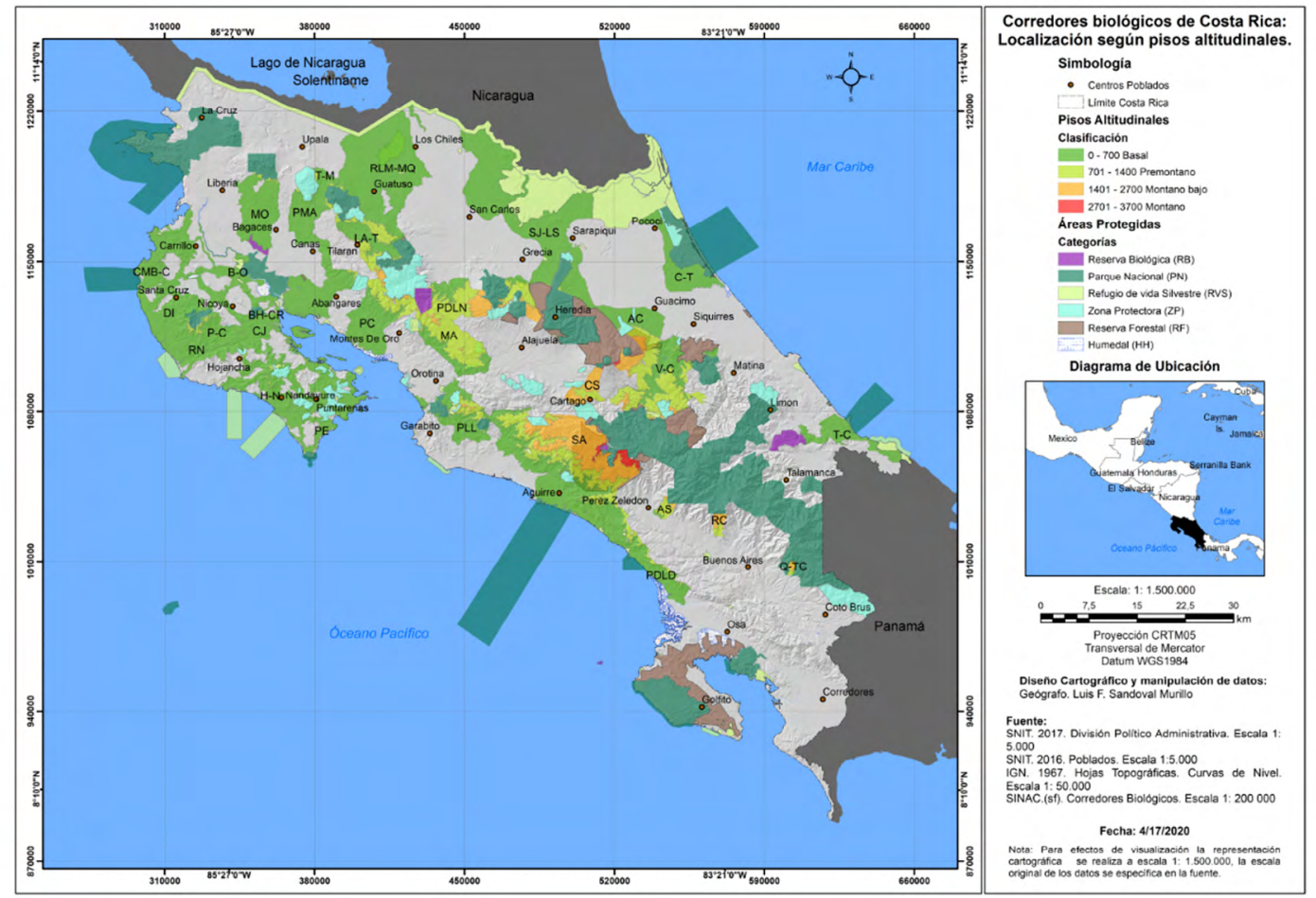

Fuente: Elaboración propia.

Lo anterior, muestra que la formulación de CB's no incorpora aspectos de estrategias de movilidad a lo largo de los diferentes pisos altitudinales como respuesta a la variabilidad climática, lo cual debería ser un aspecto prioritario para incorporarlo en propuestas como un elemento clave o como elementos prioritario para las actuales iniciativas. 
Figura 6. Localización de los Corredores Biológicos de acuerdo a los pisos altitudinales.

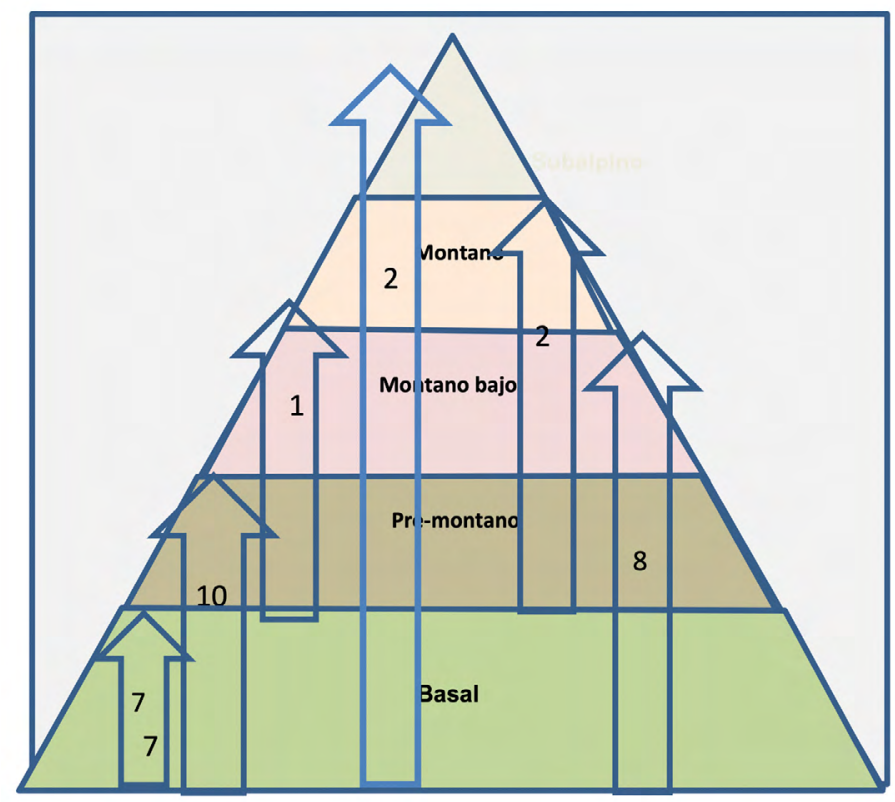

Fuente: elaboración propia

\section{Procesos de conectividad y fragmentación dentro de CB}

El establecimiento de un $\mathrm{CB}$ debería facilitar la conectividad tanto estructural como funcional, lo cual puede estar influenciado por la superficie de la cobertura natural (Morera y Sandoval, 2013).

Durante 2000-2015, $20 \%$ de los CB's redujeron su cobertura natural mientras que en la mayoría (80 \%) se incrementó la misma, siendo El Quetzal-Tres Colinas y el Paso del Mono Aullador los que presentaron mayor recuperación (Figura 7,8, 9 y 10). Un elemento que ha aportado positivamente en el mejoramiento de la cobertura natural dentro de los $\mathrm{CB}$ ha sido la priorización para la asignación del Pago por Servicios Ambientales que durante el periodo 2011-15 concentró el 52 \% (Morera, Murillo y Loría, 2017). 
Figura 7. Corredores biológicos de Costa Rica: Tasa de cambio de la vegetación (2000-2015)

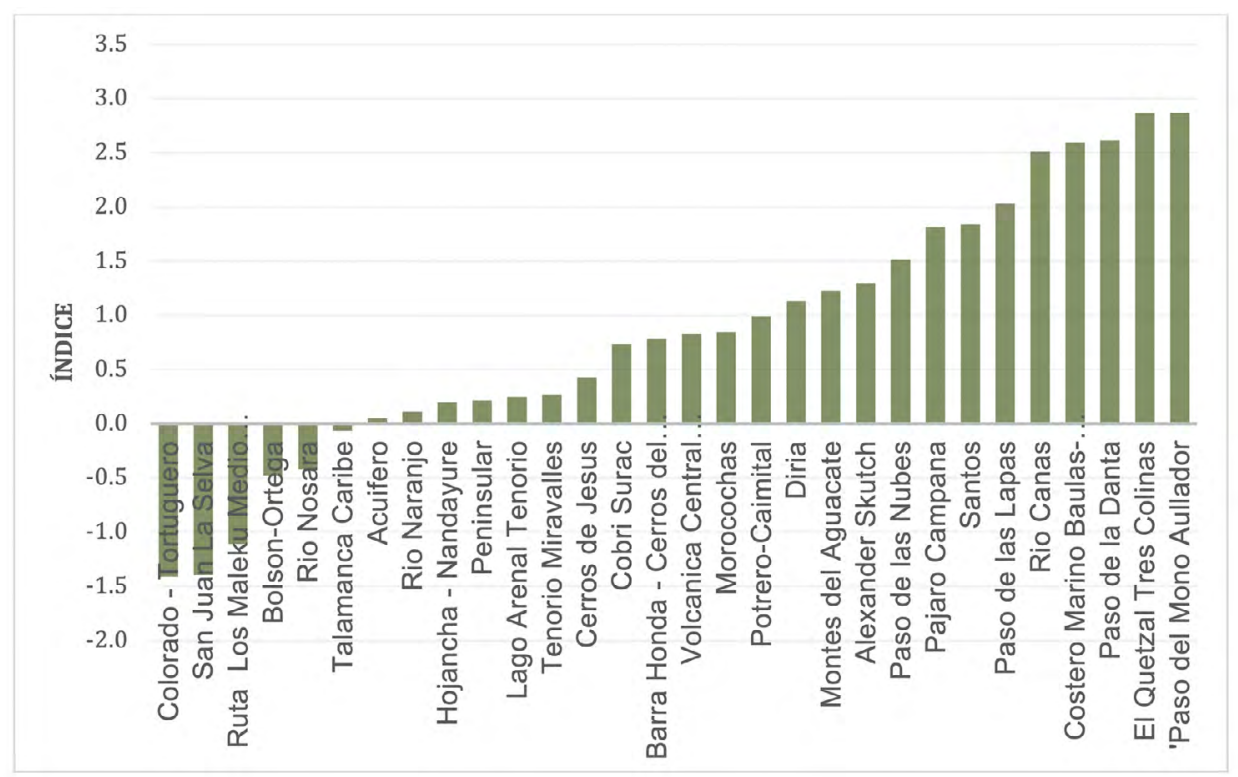

Fuente: elaboración propia

En cuanto a los procesos de fragmentación-conectividad, centrados no solo en el nivel de la cobertura natural sino en otros factores como: distancia media de los fragmentos y número de fragmentos, se encontraron resultados diferentes a los anteriores como lo hace la fórmula de IFC.

Partiendo de la premisa que el establecimiento de los $\mathrm{CB}$, deber mejorar el proceso de conectividad dentro de estos espacios, sin embargo entre 2000 y $2015,80 \%$ de los CB's aumentaron la fragmentación y solo 6 CB's (Bolsón-Ortega, Talamanca-Caribe, Rio Nosara, Colorado-Tortuguero, Ruta Los Maleku-Medio Queso y San Juan- La Selva) incrementan los procesos de conectividad (Figura 11). 
Carlos Morera-Beita Luis Fernando Sandoval-Murillo Luis Diego Alfaro-Alvarado Assessment of biological corridors in Costa Rica: landscape structure and connectivity-fragmentation processes.

Figura 8. Mapa de los Corredores Biológicos de Costa Rica: Índice de Fragmentación-Conectividad (2000)

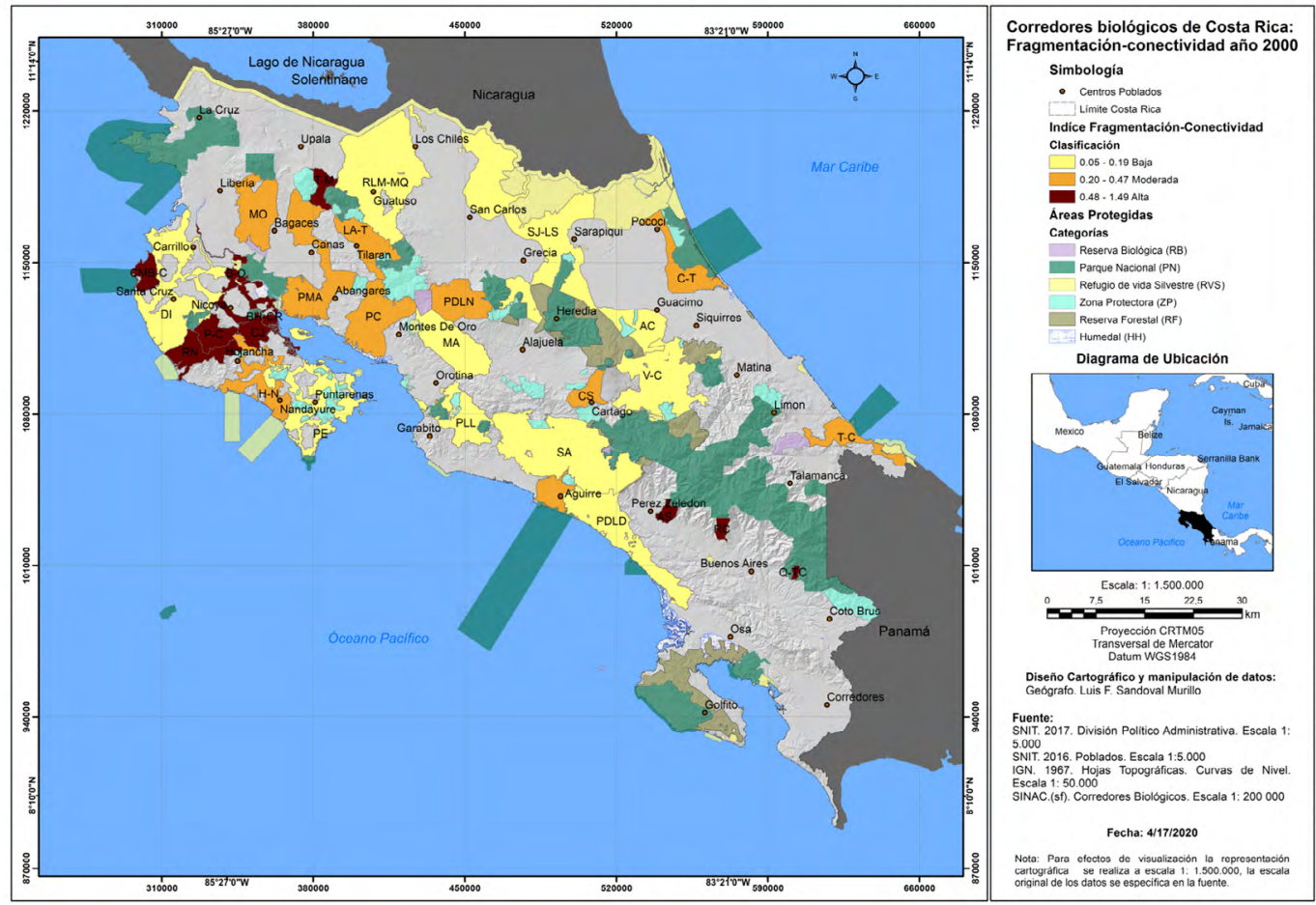

Fuente: Elaboración propia. 
Carlos Morera-Beita Luis Fernando Sandoval-Murillo Luis Diego Alfaro-Alvarado

Evaluación de corredores biológicos en Costa Rica: estructura de paisaje y

procesos de conectividad-fragmentación.

Figura 9. Mapa de los Corredores Biológicos de Costa Rica: Índice de Fragmentación-Conectividad (2015).

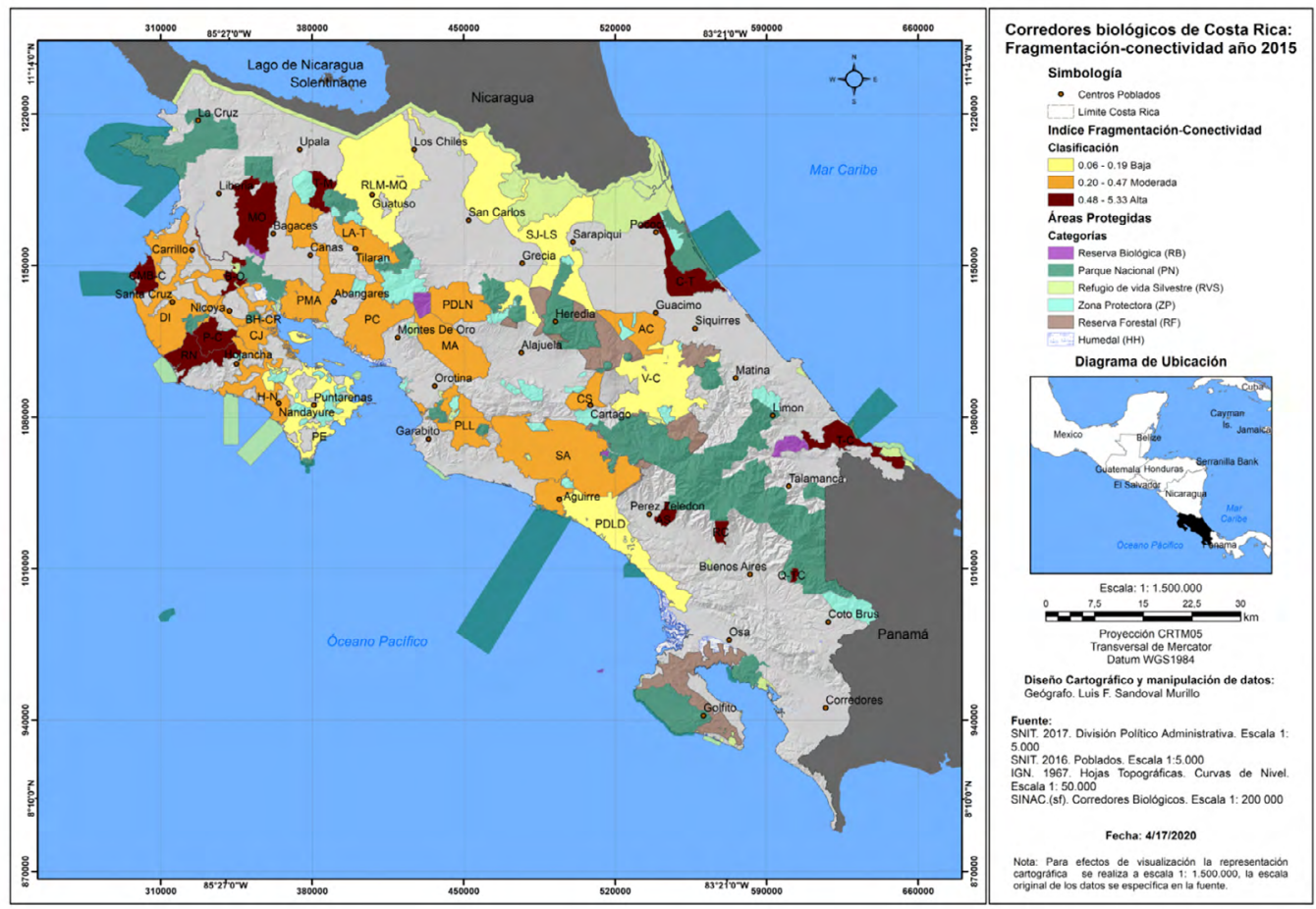

Fuente: Elaboración propia. 
Carlos Morera-Beita Luis Fernando Sandoval-Murillo Luis Diego Alfaro-Alvarado Assessment of biological corridors in Costa Rica: landscape structure and connectivity-fragmentation processes.

Figura 10. Mapa de los Corredores Biológicos de Costa Rica: Índice de Fragmentación-Conectividad, cuadro comparativo (2000-2015)

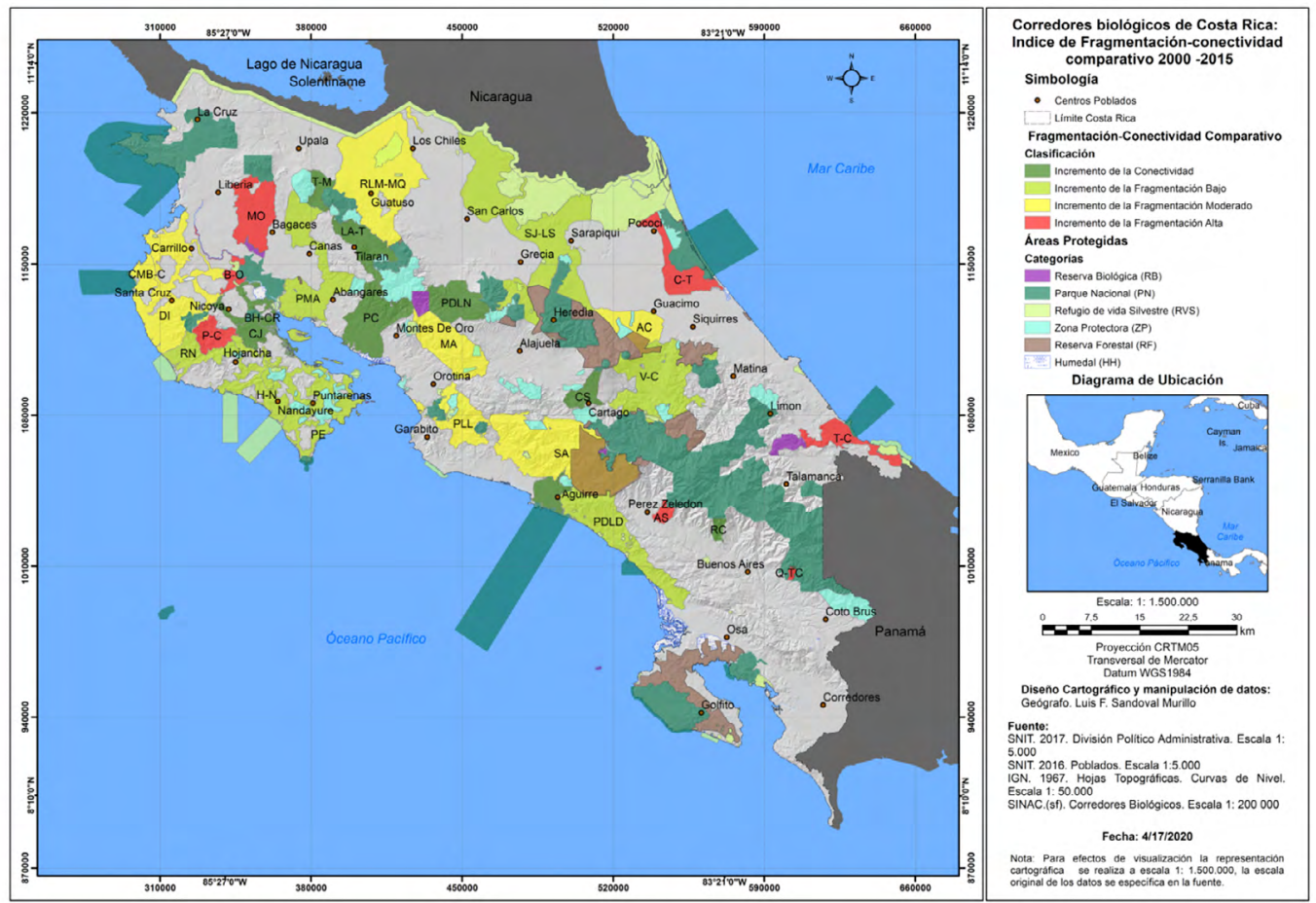

Fuente: Elaboración propia 
Figura 11. Corredores biológicos de Costa Rica, 2019: Comparación de los procesos de fragmentación-conectividad durante 2000-2015.

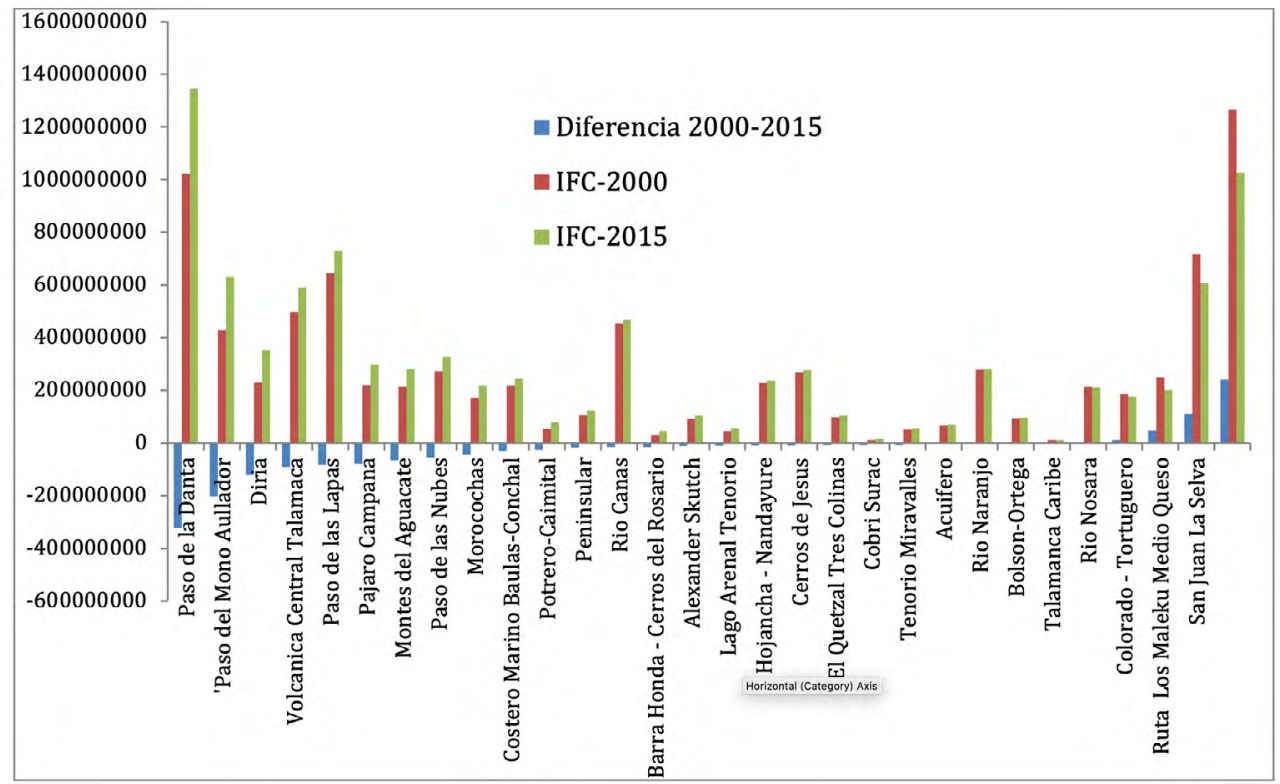

Fuente: elaboración propia

La mayoría de las iniciativas de $\mathrm{CB}$ han deteriorado sus procesos de conectividad, una vez establecidos, por lo cual se requiere implementar planes de gestión orientados a mejorar la conectividad por medio de una regeneración dirigida espacialmente. Asimismo, para consolidar los CB como un espacio efectivo de conectividad es necesario coordinar acciones con otras áreas protegidas debido a que estas áreas están articuladas.

\section{Conclusiones}

En Costa Rica, igual que muchos países, el establecimiento de los $\mathrm{CB}$ ha sido una de las principales estrategias para mejorar la conectividad de los ecosistemas naturales, lo cual toma relevancia con la variabilidad climática. El país en 2006 estableció oficialmente el Programa Nacional de Corredores Biológicos, contando para 2019 con 40 CB's. Sin embargo, solo el $75 \%$ cumple con los requisitos oficiales para declararlo como CB. Asimismo, dentro de las categorías oficiales de conservación del SINAC 
no incluyen los $\mathrm{CB}$, lo cual erosiona su adecuada gestión en comparación con otras espacios como refugios de vida silvestre y humedales, que aunque son propiedades no estatales, el estado realiza acciones más específica para su conservación ecológica.

Aunque, no se cuenta con un marco teórico consistente que respalde las iniciativas de $\mathrm{CB}$ y que la experiencia de gestión de estos son limitadas, $36 \%$ del territorio continental del país se encuentra dentro de un CB. Esta investigación evidencia la heterogeneidad en cuanto a superficie, función ecológica, estructura del paisaje, niveles de conectividad-fragmentación así como la localización de los $\mathrm{CB}$ en cuanto a pisos altitudinales como espacios de desplazamiento para la adaptación al cambio climático.

El tamaño de un $\mathrm{CB}$ es fundamental porque determina las especies que transitan o habitan este espacio, sin embargo, este aspecto no se ha incorporado como un elemento estratégico en el establecimiento o la gestión de estas áreas. Por lo cual, es urgente incorporar especies claves o metas para cada iniciativa como un aspecto que justifique su área.

En cuanto a la estructura del paisaje, la mayoría de los CB forman parte de una red integrada con otras áreas protegidas o presentan una forma de franja, lo cual aborda estos espacios como un mosaico que en algunos casos se integra a otros $\mathrm{CB}$, por lo que es necesario implementar acciones coordinadas.

La funcionalidad ecológica mostró una confusión conceptual sobre el principio de conectividad, donde algunos CB son zonas de amortiguamiento y la mayoría se localiza sobre espacios fragmentados, lo cual refuerza la necesidad de contar con estrategias de restauración ecológica localizadas estratégicamente.

Por otro lado, es necesario establecer criterios técnicos que permitan categorizar los CB para justificar su priorización, especialmente si se relaciona con los procesos de fragmentación-conectividad que aunque se haya mejorado los niveles de cobertura natural, la mayoría de los CB han incrementado los procesos de fragmentación durante los años en estudio.

Únicamente dos CB comprenden todos los pisos altitudinales presentes en el país, por lo cual el establecimiento de CB como espacios de adaptación al cambio climático, no ha sido un aspecto relevante en la identificación de estos. 
Carlos Morera-Beita Luis Fernando Sandoval-Murillo Luis Diego Alfaro-Alvarado

Evaluación de corredores biológicos en Costa Rica: estructura de paisaje y

procesos de conectividad-fragmentación.

La anterior investigación evidenció que aunque se cuenta con abundantes $\mathrm{CB}$, aún está pendiente evaluar su funcionalidad que permita justificar políticas de priorización orientadas a su consolidación. Lo cual obliga a mejorar los requisitos de establecimiento de $\mathrm{CB}$, incorporando algunas estrategias dirigidas a justificar las áreas así como estrategias de zonificación que orienten acciones de restauración.

\section{Referencias}

Acuña, E., Molina, N. \& Rodríguez, S. (2017). Análisis de la estructura del paisaje en el corredor biológico Alexander Skutch, Pérez Zeledón, en los años 2005, 2012 y 2016. Tesis. Escuela de Ciencias. Universidad Nacional, Heredia.

Alarcón, D., Anzueto, A., Galindo, A., García, J., Gómez, C., Porras, L. \& Sáenz, J. (2003). Evaluación de la biodiversidad en el corredor biológico: Una perspectiva integral de paisaje. MINAE, INBIO, The Nature Conservancy y Maestría en Manejo de Vida Silvestre. Heredia, Costa Rica.

Bosselmann, A. S. (2012). Mediating factors of land use change among coffee farmers in a biological corridor. Ecological Economics, 80, pp. 79-88.

Buzai, G. D., Humacata, L., Lanzelotti, S. L., Montes Galbán, E., \& Principi, N. (2019). Teoría y métodos de la Geografía Cuantitativa. Libro 2: Por una Geografía empírica. Instituto de Investigaciones Geográficas, Universidad de Lujan. Argentina.

Calvo-Obando, A. J. (2009). Determinación de Índices de Fragmentación y Modelamiento de la Conectividad en los Corredores Biológicos de Costa Rica. (Tesis Ingeniería Forestal). TEC. Cartago.

Chinchilla, R. (2015). Conservación y manejo integral a través del análisis de uso de la tierra y fragmentación boscosa en el corredor biológico Pájaro Campana. (Tesis). Escuela de Geografía. Universidad de Costa Rica. San José, Costa Rica.

Criado-Hernández, J. \& Marín-Cabrera, M. (2008). Conservación de la biodiversidad y desarrollo humano en bosques montanos de Costa Rica. Revista Tecnología en Marcha, 21(1), 253-263.

de Lima Marques, A., Moura, D. C., da Costa, C. R. G., de Lima, J. A. M., de Melo Lucena, A. L., Crispim, J. F. \& de Souza, J. B. (2019). 
Spaces of Environmental Conflicts in the Buffer Zones for the Protected Area of the State Park Mata Do Pau Ferro in Areia-Paraiba. Journal of Experimental Agriculture International, 1-11

Duforet-Frebourg, N. \& Slatkin, M. (2016). Isolation-by-distance-andtime in a stepping-stone model. Theoretical population biology, 108, pp. 24-35.

Ersoy, E., Jorgensen, A. \& Warren, P. H. (2018). Identifying multispecies connectivity corridors and the spatial pattern of the landscape. $U r$ ban Forestry \& Urban Greening. vol. 40, pp. 308-322. https://doi. org/10.1016/j.ufug.2018.08.001

Ernst, B.W. (2014). Quantifying landscape connectivity through the use of connectivity response curves. Landscape Ecol, 29, pp. 963-978. doi:10.1007/s10980-014-0046-7

Fletcher Jr, R. J., Didham, R. K., Banks-Leite, C., Barlow, J., Ewers, R. M., Rosindell, J. \& Melo, F. P. (2018). Is habitat fragmentation good for biodiversity? Biological conservation, 226, pp. 9-15.

Forman, R. T. (2014). Land Mosaics: The ecology of landscapes and regions (1995), . Island Press. Ingland. pp. 217

Fung, E., Imbach, P., Corrales, L., Vilchez, S., Zamora, N., Argotty, F. \& Ramos, Z. (2017). Mapping conservation priorities and connectivity pathways under climate change for tropical ecosystems. Climatic Change, 141(1), pp. 77-92.

García, E. y Gálvez, N. (2003). La Biodiversidad de Costa Rica: Sus Causas, distribución, importancia y amenazas. Serie: Problemas Ecológicos. Estudios Generales, Universidad de Costa Rica.

Haddad, n. m., Brudvig, 1. a., Damschen, e. i., Evans, d. m., Johnson, b. 1., Levey, d. j., Orrock, j. 1., Resasco, j., Sullivan, 1. 1., Tewksbury, J. J., Wagner, S. A. and Weldon, A. J. (2014). Potential Negative Ecological Effects of Corridors. Conservation Biology, 28, pp. 11781187. doi:10.1111/cobi.12323.

Heller, N.E. and Zavaleta, E. S. (2009). Biodiversity management in the face of climate change: a review of 22 years of recommendations. Biological Conservation, 142, pp. 14-32.

Hilty, J. A., Keeley, A. T., Merenlender, A. M. \& Lidicker Jr, W. Z. (2019). Corridor Ecology: Linking Landscapes for Biodiversity Conservation and Climate Adaptation. Island Press. England 
Hilty, L. M., Arnfalk, P., Erdmann, L., Goodman, J., Lehmann, M. \& Wäger, P. A. (2006). The relevance of information and communication technologies for environmental sustainability-a prospective simulation study. Environmental Modelling \& Software, 21(11), pp. 1618-1629.

Horskins, K., Mather, P.B. \& Wilson, J.C. (2006). Corridors and connectivity: when use and function do not equate. Landscape Ecol, 21, p. 641. https://doi.org/10.1007/s10980-005-5203-6

Kappelle, M. (2016). Costa Rican Ecosystems. University of Chicago Press.

Keeley, A.T.H., Beier, P. \& Gagnon, J.W. (2016). Landscape Ecol., 31, pp. 2151. https://doi.org/10.1007/s10980-016-0387-5

Khazan, E. S. (2014). Tests of biological corridor efficacy for conservation of a Neotropical giant damselfly. Biological conservation, 177, 117-125.

Krosby, M., Tewksbury, J., Haddad, N. M. \& Hoekstra, J. (2010). Ecological connectivity for a changing climate. Conservation Biology, 24(6), pp. 1686-1689.

Lewis, S. L., y Maslin, M. A. (2015). Defining the anthropocene. Nature, 519 (7542), p. 171.

Lochran, W. Traill, Corey, J. A. Bradshaw, Barry W. Brook. (2007). Minimum viable population size: A meta-analysis of 30 years of published estimates. Biological Conservation, 139(1-2), pp. 159-166.

McClure, M. L., Hansen, A. J., \& Inman, R. M. (2016). Connecting models to movements: testing connectivity model predictions against empirical migration and dispersal data. Landscape Ecology, 31(7), pp. 1419-1432.

Morera, C. \& Nel-lo, M. (2017). Local Level Policies for Tourism Management in Protected Areas: Experiences from Costa Rica (Chapter 6). In: Protected Areas: Policies, Management and Future Directions. (Editors: Sharif Ahmed Mukul and A. Z. M. Manzoor Rashid). Nova Science Publishers. New York, USA.

Morera ,C., Murillo, L.F. y Loría A. (2017). Dinámica espacio-temporales de los Servicios Ambientales (PSA) en Costa Rica 2011-2015; Memoria Encuentro de Geógrafos de América Latina. La Paz, Bolivia. 
Morera, C. y Sandoval, L. F. (2018). Fragmentación y conectividad de la cobertura natural a nivel cantonal en Costa Rica durante los años 2000 y 2015. Revista Geográfica de América Central, Edición 61e (4). Especial I Congreso Ciencias de la Tierra y el Mar. Universidad Nacional.

Morera, C. y Sandoval, L. F. (2015). Aplicabilidad de indicadores de estructura de paisaje para evaluar transformaciones en escenarios tropicales. En: Métodos y Técnicas Geográficas Aplicadas al Ambiente: Una Visión Latinoamericana. Universidad Autónoma de San Luis Potosí, México. EUNA, Heredia.

Morera, C. \& Sandoval, L. F. (2016). Evaluación de la cobertura boscosa en Costa Rica: un análisis a nivel de áreas de conservación en el año 2000. Revista Geográfica de América Central, 2(56), pp. 163-182.

Morera, C. y Sandoval, L. (2013). Los procesos de fragmentación y la conectividad forestal en Costa Rica: Dos casos de estudios. En: Geografía, Paisaje y Conservación. ECG - UNA.

Moss, M. (2001). Landscape Ecology Applied in Land Evaluation. Development and Conservation. ITC, 81

Organización de las Naciones Unidas para la Alimentación y la Agricultura (FAO) (1996). Forest Resources Assement. (Global Symthesis. FAO Forestry Paper No.124). Rome, Italy.

Pfaff, A. S., \& Sánchez-Azofeifa, G. A. (2004). Deforestation pressure and biological reserve planning: a conceptual approach and an illustrative application for Costa Rica. Resource and Energy Economics, 26(2), 237-254.

Rempel, R., Carr, A. \& Elkie, P. (2008). Patch analyst for ArcGIS®. Centre for Northern Forest Ecosystem Research, Ontario Ministry of Natural Resources. Lakehead University.

Rosenberg, D. K., Noon, B. R., \& Meslow, E. C. (1997). Biological corridors: form, function, and efficacy. BioScience, 47(10), 677-687.

Solís, V., Madrigal P., Cruz I. \& Fonseca M. (2002). Mesoamerican Biological Corridor and local participation: Parks [Parks]. Vol. 12, no. 2, pp. 42-54. 
Stewart, J. S., Wang, L., Lyons, J., Horwatich, J. A. \& Bannerman, R. (2001). Influences of watershed, riparian-corridor, and reach-scale characteristics on aquatic biota in agricultural watersheds 1. jawra Journal of the American Water Resources Association, 37(6), pp. 1475-1487.

Taubert, F., Fischer, R., Groeneveld, J., Lehmann, S., Müller, M. S., Rödig, E. \& Hutch, A. (2018). Global patterns of tropical forest fragmentation. Nature, 554(7693), p. 519.

Vergara, P.M. (2011). Matrix-dependent corridor effectiveness and the abundance of forest birds in fragmented landscapes. Landscape Ecol 26, pp. 10-85 doi:10.1007/s10980-011-9641-z

Villate, L. Canet-Desanti, O. Chassot, G. \& Monge-Arias. (2009). Una estrategia exitosa de conservación., CATIE, Costa Rica.

Worboys, G., Francis, W. L. \& Lockwood, M. (Eds.). (2010). Connectivity conservation management: a global guide (with particular reference to mountain connectivity conservation). Earthsca

Yang, L., Liu, B., Zhang, Q., Zeng, Y., Pan, Y., Li, M. \& Lu, Y. (2019). Landscape structure alters the abundance and species composition of early-season aphid populations in wheat fields. Agriculture, Ecosystems \& Environment, 269, pp. 167-173. 
